\title{
La industria de la información: explosión tecnológica y revolución económica
}

\author{
José Luis González-Rubio \\ Subdirector de Nuevos Negocios \\ de Multivisión
}

El PRESENTE documento expone un panorama general sobre las transformaciones recientes en la industria de la información desde la perspectiva empresarial y propone reflexionar sobre la función del comunicador ante estos cambios.

En la primera parte se presentan algunos ejemplos de la recomposición de la industria de las telecomunicaciones como consecuencia de la evolución tecnológica y la liberación de la competencia. Enseguida se analiza la relevancia de la privatización del sector telecomunicaciones en México y se expone el crecimiento de la industria,generado por el programa de modernización emprendido recientemente. Finalmente se expone la necesidad de adaptar la profesión del comunicador a las exigencias que la nueva conformación de la industria reclama.

\section{La industria de la información: catalizador del desarrollo}

Las épocas de desarrollo económico son identificadas por la relevancia de un determinado sector que imprime la dirección y el ritmo del crecimiento. En los

1 El presente documento sintetiza la ponencia preparada para el coloquio "Nuevas perspectivas del comunicador", organizado por la Universidad Veracruzana y Diario de Xalapa, y celebrado del 16 al 19 de noviembre de 1994 en la ciudad de Xalapa, Veracruz.

La repentina modificación de la expectativas económicas a partir del 19 de diciembre de 1994 enmarca en un contexto económico distinto los supuestos en que se sustenta esta propuesta. Es razonable anticipar que la desaceleración económica retrasará el ritmo de crecimiento previsto; sin embargo, la tesis que postula la necesidad de la expansión de las telecomunicaciones como piedra angular del desarrollo nacional, continúa vigente. 
inicios del siglo XX el desarrollo encontró su expansión en el intercambio de bienes en la tecnología del transporte ferroviario. Posteriormente, en la época de la posguerra, el impulso de la reconstrucción económica se sustentó en la industria de la transformación y en la década de los ochenta las fuerzas motrices de la economía se trasladaron del sector industrial al sector terciario, en un intento por aligerar estructuras demasiado onerosas.

Sin embargo, en virtud de las aceleradas transformaciones tecnológicas, en la presente década la orientación de las inversiones parece indicar que el crecimiento económico será conducido por el desarrollo de la industria de la información, conformada, tal como la definiera Koji Kobayashi, por la integración sinérgica de las tecnologías de la computación, las comunicaciones y los componentes. ${ }^{2}$

La industria de la información ha incidido en una transformación directa de la naturaleza de la economía. La convergencia aventurada de las industrias de telecomunicaciones, computación y radiodifusión permite prever la aparición de nuevos servicios que representan atractivas oportunidades de negocio, explotando la capacidad de transmitir información en forma inmediata e interactiva a través de grandes distancias.

Los sectores de actividad centrados en la transmisión de información están llamados a constituir el núcleo de la actividad económica en el futuro inmediato. Un análisis del crecimiento económico por rama de actividad conduce a concluir, de acuerdo con William Burger (1994), que: “(en los últimos años)... las industrias que registran crecimiento están orientadas al movimiento de imágenes, información y servicios. Este sector será el que tendrá un beneficio desproporcionado en esta (nueva) organización social". La industria derivada del desarrollo de tecnologías de información está llamada a constituir uno de los campos de desarrollo más dinámicos en la próxima década.

En Estados Unidos, desde 1983, la industria de la información absorbe la mayor proporción de inversiones en tecnología. En el período comprendido entre 1975 y 1993, del total de la inversión efectuada en este país, la destinada a maquinaria - fundamento tradicional de los activos del sector industrial - registró un descenso de $32 \%$ a $18 \%$. Inversamente, la inversión destinada a equipamiento alimentado por información, incluido equipo de cómputo, creció de $17 \%$ en 1975 a casi $50 \%$ en 1993. Mandel (1994: 36-39).

2 Citado por Alfredo Pérez de Mendoza (1993). 
Figura 1:

Destino de la inversión privada en equipamiento

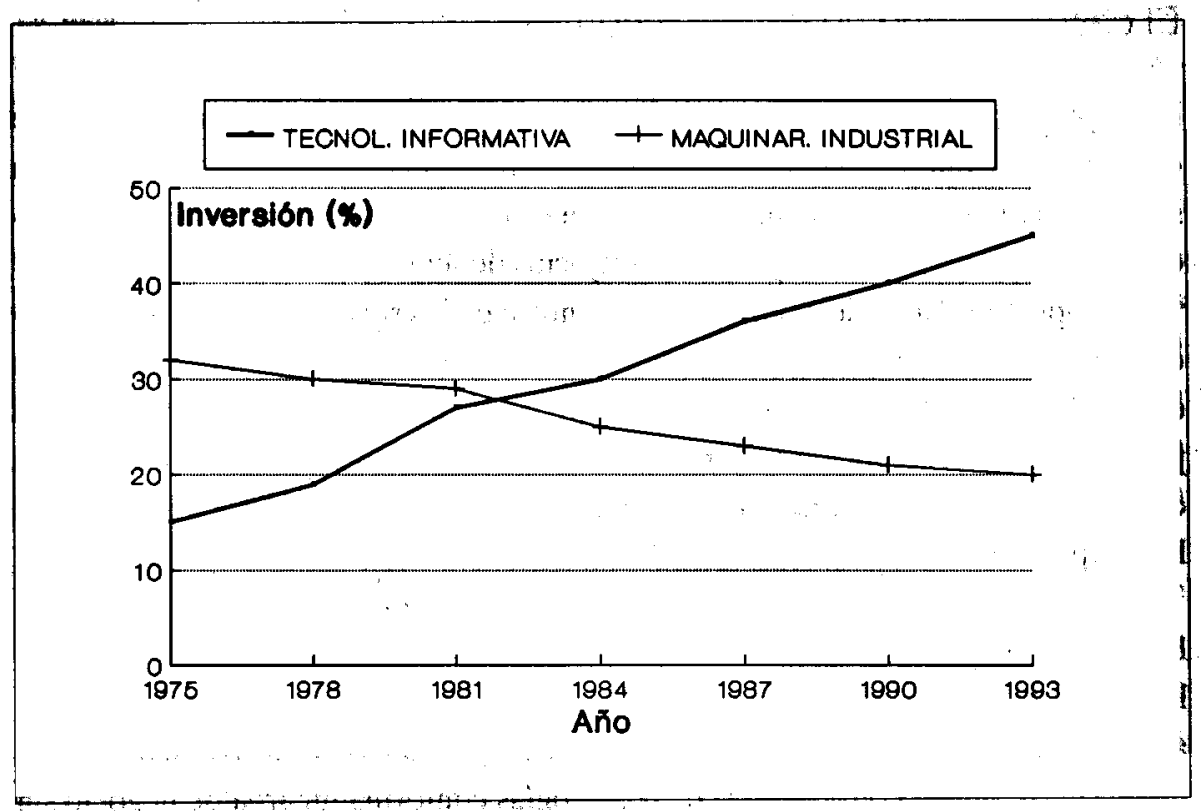

Fuente: International Business Week, 13-6-94.

El mismo fenómeno de crecimiento del sector de la información se advierte en cuanto a la participación de equipo de cómputo y telecomunicaciones. En 1980 las exportaciones estadounidenses en este rubro representaban el $7 \%$ del total, la misma proporción que las de la industria de la aeronáutica - ramo líder en la economía de Estados Unidos. Sin embargo trece años después, en 1993, las exportaciones de equipamiento clasificado como tecnología de información y telecomunicaciones - sin contar software - ascendieron a 62 mil millones de dólares, cifra que representa casi el doble de las ventas en el exterior de la industria aeronáutica ese mismo año (33 mil millones de dólares). Mandel (1994: 36-39).

Este rápido crecimiento de la participación de actividades sustentadas en el manejo de información es indicativo de la creciente incidencia del sector en los procesos productivos de la economía de este fin de siglo. 


\section{Innovación tecnológica y expectativas económicas}

\section{El caso europeo: inversión y competencia}

El impulso proporcionado por la desregulación se sustenta en un importante cambio en las políticas de desarrollo, consistente en privilegiar la inversión privada sobre la vía del financiamiento público. En este marco la industria de la comunicación constituye una de las áreas de negocios de mayor atractivo.

Las oportunidades de negocio se concentran en los sectores de las telecomunicaciones y el entretenimiento. El desmantelamiento de los monopolios telefónicos estatales y la disminución de cuotas de producción nacional en televisión, han provocado la redefinición del rol de las telecomunicaciones en virtud de la convergencia de dos fuerzas: la aparición de la tecnología digital y la liberación de la competencia.

La sinergia de tecnologías ha conducido a la alianza de corporaciones que se habían desarrollado por tecnologías específicas. Las alianzas se multiplican ante las grandes expectativas de retorno de la inversión. En Gran Bretaña se desarrollan alianzas estratégicas entre compañías telefónicas y sistemas de televisión por cable, de tal suerte que la convergencia de tecnologías conforma un área de negocio sin precedentes en la historia de las telecomunicaciones. Entre las compañías que han incursionado en Europa se encuentran firmas como Nynex, Southwestern Bell, MCI Communications, Sprint, AT\&T, así como Cox Cable, Comcast, International Cable-Tel, Jones Cable y Pacific Telesis.

Hasta mediados de la década de los noventa, las legislaciones nacionales prohíben la oferta de servicios telefónico y televisivo por una misma compañía o en una misma región como es el caso en Estados Unidos y México. Sin embargo, en la Gran Bretaña esta convergencia no está excluida, lo que ha provocado la aparición de nuevos servicios. US West y Southwestern Bell han aprovechado las sinergias ofrecidas por el marco regulatorio para ofrecer a través del mismo cable servicios de televisión y de telefonía (Frankel, et al, 1993: 26-31). Se ofrecen incluso servicios nuevos como consultas a bibliotecas a través de redes de televisión por cable/teléfono.

British Telecommunications, en asociación con operadores de televisión por cable planea invertir 37 mil millones de dólares en los próximos seis años en redes que conducirán video, voz y datos a hogares y oficinas (Edmondson, 1994). La 
inversión programada para los próximos cinco años por algunas Baby Bells, en asociación con operadores de cable suma alrededor de 9 mil millones de dólares. El destino de estas inversiones se concentrará en el desarrollo de sistemas de televisión por cable, redes telefónicas y sistemas celulares, principalmente en Gran Bretaña, pero también en Francia, Escandinavia y Europa del Este (Rapaport, 1993: 36-40).

Las cuantiosas inversiones anunciadas originarán una gran recomposición de la industria. Algunos expertos estiman que entre 1995 y el año 2000, los países integrantes de la Unión Europea recibirán inversión por 76 mil millones de dólares - más de lo que se ha gastado en los últimos 115 años, desde la invención del teléfono - para ofrecer nuevas formas de comunicación, entre las que se cuentan redes avanzadas, video interactivo, exhibición cinematográfica en compresión digital y otros servicios como juegos multimedia, entretenimiento y educación (Edmondson, 1994).

En conjunto se calcula que el mercado de telecomunicaciones encierra un potencial de 297 mil millones de dólares hacia el año 2000, cifra que representará el 4.5\% del PIB del la Unión Europea, rebasando incluso la participación de la industria automotriz (Frankel et al, 1993: 26-31). Los efectos de estos cambios acaso no serán descifrables sino en el mediano plazo.

El mercado europeo ofrece asimismo un panorama atractivo para la industria del entretenimiento: NBC - que difunde su señal en Europa a través de Europe's Super Channel - QVC, CMT (Country Music Television) y Discovery Channel incursionan en las audiencias europeas, soportados por fuertes inversiones (Rapaport, 1993: 36-40).

Otros ejemplos de nuevas dinámicas en el campo del entretenimiento y la diversificación de mercados son la asociación de TNT con Cartoon Network y la incursión en Europa de Viacom Enterprises, The Family Channel y Sci-Fi Channel. MTV, el más grande canal musical de Estados Unidos cuenta con más suscriptores fuera que dentro de ese país: 56.5 millones de abonados. En 1993 sus ingresos ascendieron a más de 70 millones de dólares y se estima que hacia finales de 1994 sumarán más de 100 millones de dólares (Rapaport, 1993).

La revolución tecnológica aunada a las grandes inversiones requeridas ha planteado la reorientación de los tradicionales negocios de comunicaciones. El caso de ITT es revelador de la voluntad de aprovechamiento de sinergias que ofrece el sector. Recientemente ITT hizo pública su intención de vender su división 
financiera - valuada en 3 mil millones de dólares - para invertir en la industria del entretenimiento, posiblemente mediante la participación en una network, que podría ser NBC O CBS. ITT ya ha concretado parcialmente su incursión en el negocio del espectáculo con la adquisición del Madison Square Garden de Nueva York en asociación con Cablevision Systems Corp., operación valuada en 1,080 millones de dólares. $^{3}$

Las telecomunicaciones y el entretenimiento se revelan como una de las ramas más atractivas para la inversión. La rápida evolución de las tecnologías en los últimos años y el desarrollo que puedan tener los nuevos servicios dificulta prever el futuro.

Sin embargo resulta razonable anticipar que el auge de las telecomunicaciones será la causa de un impacto de gran aliento sobre el desarrollo de las economías. $\mathrm{El}$ acelerado desarrollo de infraestructura y la aparición de nuevas aplicaciones en los países postindustrializados acentuará la brecha con los países en desarrollo. No obstante, éstos últimos parecen estar empeñados en reducir las distancias mediante el impulso a la modernización que pretenden imprimir para realizar, en condiciones más favorables, su ingreso en el nuevo siglo.

\section{Países en desarrollo: los imperativos de la modernización}

Algunos países tradicionalmente clasificados como pertenecientes al mundo en desarrollo han advertido la imperante necesidad de modernizar su infraestructura de telecomunicaciones como condición para ingresar en una etapa superior de desarrollo.

El rezago tecnológico de países de América Latina, Asia o Europa del Este representa claramente un obstáculo para el crecimiento. Mediante la aplicación de tecnologías convencionales, se requerirían varios años para superar el retraso. Sin embargo, algunas tecnologías - fibra óptica, tecnología digital, sistemas de transmisión vía wireless - ofrecen opciones para atraer inversiones y acelerar su recuperación.

3 Ver: "ITT venderá su división financiera para entrar en cadenas de televisión". (1904): Reforma, 19 de septiembre, p. 28A. Se trata de la traducción de un artículo de Molly Baker (s. f.): The Wall Street Journal (s. d.). 
Algunos ejemplos de lo que podría ser la nueva conformación del mundo en cuanto a infraestructura de telecomunicaciones parecen sugerir una voluntad de acercamiento - tecnológico y económico - con países desarrollados.

Un prototipo de esta voluntad de saltar etapas para incorporarse al desarrollo en telecomunicaciones lo constituye la República Popular China, que para el año 2000 planea instalar 80 millones de líneas telefónicas, con lo cual prácticamente cuadruplicará sus líneas en servicio. Esta meta implica agregar más de 12 millones de líneas cada año - Estados Unidos agrega 5 millones de líneas por año. Para modernizar su sistema de telecomunicaciones - que incluye la instalación de fibra óptica, estaciones satelitales y redes de comunicación wireless - las autoridades chinas planean efectuar inversiones por 100 mil millones de dólares en los próximos 10 años (Frankel et al, 1993: 28).

El mismo fenómeno de modernización se advierte en países como Tailandia, Vietnam y en general en los países del sureste asiático. Con una inversión de 150 mil millones de dólares en los próximos cinco años estos países planean invertir en telecomunicaciones tres veces más que lo invertido en la década de los ochenta (McGrath, 1994: 16-35).

Otro ejemplo de la revaloración de las telecomunicaciones como condición fundamental para la incorporación al desarrollo lo protagoniza el gobierno de la India. Como parte de un proyectode modernización elgobierno hindú ha anunciado su intención de efectuar inversiones en los próximos tres años, por más de 7,700 millones de dólares a fin de comunicar regiones rurales del país (McGrath, 1994).

La voluntad de modernización se repite en países del este europeo, donde se han diseñado innovadores esquemas de asociación para materializar proyectos de inversión. En Hungría, por ejemplo, el gobierno concretó una asociación de la telefónica nacional y Deutsche Bundespost Telekom de Alemania/Ameritech International Inc; éstos últimos participan como socios tecnólogos con $30 \%$ del capital. Como complemento, el gobierno húngaro ha otorgado concesiones a inversionistas extranjeros para construir un sistema de comunicación celular digital de alta tecnología.

Estas propuestas de negocio son indicativas de que el sector de telecomunicaciones constituye un catalizador para el desarrollo y al mismo tiempo se convierte en el común denominador de la expansión y el crecimiento. Sin embargo, es razonable prever que la modernización - la recuperación del rezago telefónicoy aún la propuesta de innovaciones, como las nuevas aplicaciones de la interacti- 
vidad, se concentrarán en aquellas economías que encierren la capacidad de crear los mercados que las soporten.

La dificultad de la aplicación tecnológica para impulsar el desarrollo en un entorno de rezago económico es enfrentada asimismo, por algunos países de América Latina, donde la dinámica del sector comunicaciones es igualmente explosiva.

La venta de las compañías telefónicas de Argentina, México, Venezuela, Chile y Perú son prueba de ello. En este último país, el Estado enajenó recientemente un paquete accionario representativo del $35 \%$ del capital de la compañía telefónica nacional a un grupo integrado por Telefónica de España, en asociación con inversionistas nacionales y extranjeros; el paquete accionario, que incluye la participación en la compañía cie teléfonos y en el monopolio de larga distancia Entel, fue valuado en 2 mil millones de dólares. La estrategia empresarial se orienta a explotar las sinergias ofrecidas: la red de TV Panamericana y Radio Programas del Perú, junto con la red de televisión TeleCable participan igualmente en el negocio de la telefonía celular (Bowen, 1994: 29-30).

La nueva conformación tecnológica y empresarial del sector conduce a anticipar que en un futuro próximo, la convergencia de tecnología y la homologación de normas ofrecerán amplias perspectivas de inversión en nuevos servicios que serán rápidamente integrados a la vida doméstica y profesional.

En términos generales el atractivo del negocio que encierra el sector telecomunicaciones explica la aparición de audaces propuestas de inversión en el mundo entero. En caso de concretarse algunos proyectos de comunicación celular vía satélite, la industria de la transmisión de la información se transformará radicalmente; entre ellos se cuenta el proyecto Iridium - promovido por Motorola -consistente en el lanzamiento y operación de 66 satélites mediante una inversión de 3,500 millones de dólares. Existen otros proyectos similares para instalar lo que sería una red mundial de telecomunicaciones. Entre ellos destacan el proyecto promovido por Inmarsat, que implica inversiones por 2,500 millones de dólares; Globalstar de Loral/Qualcomm, con 1,800 millones de dólares, y el ambicioso proyecto de Microsoft/McCaw Cellular, que propone lanzar 840 satélites de comunicación para el año 2001 con el fin de comunicar el planeta entero con tecnología celular, mediante una inversión de 9 mil millones de dólares (McGrath, 1994). 
En el contexto internacional, no solamente en el mundo industrializado, sino también en los países en desarrollo, se advierte una amplia revolución de la industria de la información, que trastoca las referencias tradicionales de la materia. El desarrollo tecnológico, respaldado por fuertes capitales, así como la creciente desregulación, postulan nuevas formas de concebir la disciplina de la comunicación. Estos cambios provocarán repercusiones que, al igual que en el resto del mundo, en México transformarán el panorama de la industria.

\section{Nuevos escenarios de la comunicación: el caso de México}

\section{Inicio de un nuevo ciclo}

Cuando como parte de su programa de desincorporación de empresas no estratégicas ni prioritarias, el gobierno mexicano anunció su intención de poner a la venta sus intereses en Teléfonos de México y en la televisión estatal, en realidad inauguraba una nueva etapa en el campo de las comunicaciones. Al tiempo que otorgaba nuevas concesiones para la oferta de diversos servicios, redefinía la relación del Estado con la industria, promovía la competencia entre inversionistas privados y propiciaba la modernización tecnológica indispensable para recuperar el rezago de las telecomunicaciones en el país.

La privatización más importante del sexenio 1988-1994 fue sin lugar a dudas la de los 18 bancos múltiples, no solamente en términos de captación de recursos, sino por la trascendencia de la intermediación financiera para el desarrollo nacional.

Los ingresos del gobierno federal por la venta de los bancos representó 52 por ciento de la recaudación derivada de la venta de empresas públicas (Piz, 1994: 44). El segundo lugar en cuanto a recaudación fue ocupado por Teléfonos de México (Telmex). Los ingresos derivados de esta operación fueron de 6,180 millones de dólares, lo cual representa 26 por ciento de los ingresos recaudados por el gobierno federal en el programa de desincorporación. El tercer lugar fue ocupado por el paquete de medios de comunicación, al representar 2.7 por ciento de los ingresos.

La importancia del sector se pone de relieve al analizar las empresas de comunicación en relación con el resto de las entidades desincorporadas: las dos operaciones de desincorporación más importantes del sexenio después de la banca 
fueron precisamente las correspondientes a las empresas de comunicación del gobierno federal. Sin considerar los ingresos recaudados por la venta de los bancos, la venta de Telmex representó 54.3 por ciento de los ingresos; en tanto que la venta del paquete de medios significó 5.6 por ciento del total. Así, las empresas de comunicación representaron 60 por ciento de los ingresos recaudados por la desincorporación de instituciones no bancarias. Un análisis más detallado habrá de centrarse en la rentabilidad de estos negocios.

En cuanto a la venta de los medios de comunicación social, después de efectuar un proceso de saneamiento, el gobierno federal decidió enajenar sus intereses en televisión y en la industria cinematográfica, así como en la industria periodística. Para ello conformó un "paquete de medios de comunicación", que pondría a la venta mediante un proceso de subasta pública.

El paquete quedó finalmente integrado por las entidades que forman parte de las redes nacionales de los canales 7 y 13 de televisión, además de la Compañía Operadora de Teatros (Cotsa), que agrupaba alrededor de 260 salas de exhibición cinematográfica, los Estudios América, el Canal 2 de Chihuahua y el periódico $E l$ Nacional.

La oferta ganadora rebasó todas las expectativas al proponer un precio de 640 millones de dólares por el paquete, sin considerar el rotativo, mismo que a la postre sería retirado de la subasta. Con esta operación el gobierno federal se retiraba de la administración de empresas de televisión y de exhibición cinematográfica, manteniendo su participación en estaciones radiofónicas. La venta del paquete de medios cerraba un ciclo en la participación del Estado en la comunicación social. La privatización de las empresas de comunicación fue determinante no sólo en términos de ingresos, sino también porque acaso son las entidades que más influencia directa ejercen sobre la mayoría de los mexicanos.

En virtud del desarrollo tecnológico y de los cambios que pueden introducir en la organización social, las telecomunicaciones están llamadas a constituir una de las actividades productivas más dinámicas de la economía. Las anunciadas inversiones en el sector se inscriben en un contexto que prefigura grandes transformaciones para México en los próximos años. 


\section{La modernización}

Durante el periodo 1988-1994 el país registró una evolución sorprendente en la modernización de su infraestructura y la aparición de nuevos competidores en diferentes servicios.

Los avances registrados en este periodo no tienen precedente: casi se duplicó el número de líneas telefónicas (de 4.4 a 8.3 millones) y se cuadruplicó el número de poblaciones atendidas (6 mil a 25 mil) y el de casetas públicas (44 mil a $196 \mathrm{mil}$ ). A partir de la privatización de la telefonía y hasta 1993 la instalación de nuevas líneas ha representado un incremento compuesto de 12 por ciento anual, lo que coloca a Telmex como la compañía con el crecimiento más alto en el mundo. La densidad telefónica en el país se duplica, aunque está lejos de alcanzar los estándares de 45 líneas por cada 100 habitantes promedio de los países de la Ocde, o los de Estados Unidos y Canadá, donde se registran 56 y 59 líneas por cada 100 habitantes respectivamente (Matus, 1993: 28A).

En los últimos seis años el sector telecomunicaciones en México recibió inversiones por más de 13,500 millones de dólares, con lo que registró un crecimiento superado solamente por la industria de la construcción (Matus, 1994: 26A). Se estima que durante el próximo sexenio la iniciativa privada nacional y extranjera invertirá alrededor de 60 mil millones de dólares en el sector, el equivalente a la mitad de la deuda externa del país (Cardoso, 1994: 48).

Asimismo se expandieron otros servicios como la radiolocalización de personas (paging) que triplicó el número de usuarios; la localización de flotillas (trunking) evolucionó de mil a 40 mil usuarios; se multiplicó por 100 el número de suscriptores de telefonía celular - de 5 mil a 500 mil - igualmente se duplicaron las concesiones de televisión abierta y crecieron en 50 por ciento las de la televisión por cable; se instauró el servicio de televisión y radio restringida y el de televisión de difusión directa 'Direct to Home' (DTH) (Matus, 1994). Otros servicios que diversifican los soportes de comunicación son los servicios de valor agregado para la transmisión de datos, correo electrónico, video texto y videoconferencias.

El proceso de modernización de las telecomunicaciones se inscribe en un contexto de grandes rezagos. A pesar de ser la décimo cuarta economía más grande del mundo, México se coloca en el septuagésimo lugar en términos de densidad telefónica (SCT, s. f.), lo que es indicativo del contraste existente entre el potencial económico del país y el aplazamiento de la modernización para el conjunto de la 
población. El sector telecomunicaciones constituye un área de negocios de gran atractivo; el servicio de larga distancia es un claro ejemplo. El desarrollo de mercados se presentará en la medida en que el país logre mantener un ritmo de crecimiento acorde con las necesidades de la población.

La lectura de los cambios acelerados - creación de nuevos desequilibrios y necesidad de conjuntar conocimientos sobre la orientación de la industriaencierra un reto profesional inédito para los especialistas en comunicación.

\section{Nuevos retos profesionales}

La revolución de las telecomunicaciones ofrece nuevas perspectivas para su estudio. Es razonable prever que el sector telecomunicaciones continuará experimentando un crecimiento dinámico en los próximos años: por una parte la tecnología continuará en acelerada evolución y por otra, las perspectivas de la economía mexicana permiten prever el surgimiento de mercados que ejercerán una fuerte atracción para la inversión en el sector, lo que derivará en nuevas demandas profesionales.

Durante varias generaciones, la información ha sido evaluada, entre otras, desde la perspectiva política, sociológica o estética, desatendiendo el aspecto económico del fenómeno de transmisión de información en la sociedad industrial.

El nuevo panorama de las telecomunicaciones plantea nuevos retos para el profesional de la disciplina. Su aportación de valor agregado rebasa el inventario de medios, el análisis de sus contenidos o los efectos de su crecimiento y penetración en las sociedades.

Operaciones recientes como la alianza entre la cadena americana NBC y Televisión Azteca, la colocación en Bolsa de diversos grupos de comunicación, el inminente ingreso de inversionistas extranjeros en el mercado de la exhibición cinematográfica, la compraventa de sistemas de televisión por cable en el interior de la República, la liberación de servicios especiales y el surgimiento de nuevos sistemas de distribución de señales, conducen a extender las concepciones tradicionales del estudio de la disciplina de la comunicación. La acelerada evolución tecnológica yla consecuente modificación de infraestructuras y hábitos de consumo invitan a reflexionar sobre el objeto mismo de estudio de la disciplina.

La comparecencia de nuevos sistemas de comunicación remite ineludiblemente a la aparición de nuevas exigencias para los profesionales de la especialidad. Entre 
las más inmediatas se encuentra la urgencia de descifrar la nueva conformación del objeto de estudio y la necesidad de teorizar a partir de nuevos modelos; será deseable asimismo apoyar la formación de profesionales con orientación a las demandas empresariales por talento profesional.

El mercado profesional demandará nuevas habilidades de los especialistas en comunicación: identificar demandas insatisfechas, analizar mercados, efectuar análisis de competitividad, diseñar estrategias comerciales, efectuar estudios de oferta/demanda o establecer relaciones de costo/beneficio, entre otras. Las demandas profesionales se centrarán en el conocimiento de aspectos comerciales, administrativos, tecnológicos y financieros para el diseño y ejecución de estrategias empresariales.

En síntesis, la participación de un especialista en comunicación rebasa las concepciones tradicionales de la materia. Su contribución - en términos de valor agregado - puede ser significativa para el desarrollo de la industria. La comprensión de los fenómenos de comunicación que se producen con la concurrencia de tecnología y competencia constituyen un auténtico desafío profesional de cara a la sociedad que se conforma hoy rumbo al próximo milenio.

\section{Referencias bibliográficas}

BoweŃ Sally (1994): "Perú ya no marca ocupado". América economía Núm. 83 (mayo), pp. 29-30.

BURGER William (1994): "Brave New York". Newsweek (ed. internacional). (mayo), p. 25.

CARDoso Víctor (1994): "Prevén inversiones por más de 60 mil mdd en telecomunicaciones el próximo sexenio". La Jornada, 21 de septiembre, p. 48. México, D. F.

EDMONDSON Gail (1994): "Brave Old World". International Business Week Núm. 3361-691 (junio), pp. 36-39. 
FRANKEL Mark et al (1993): "Wiring the World". Newsweek (ed. internacional) (abril), pp. 26-31.

MANDEL Michael J. (1994): “The Digital Juggernaut". International Business Week Núm. 3361-691 (junio), pp. 36-39.

MATUS María Fernanda (1993): “Avanza telefonía celular”. Reforma, 8 de agosto, p. 26A. México, D. F.

(1994): "Telecomunicaciones. Golpean en forma suave a Telmex y Televisa". Reforma, 30 de septiembre, p. 26A. México, D. F.

MC GRATH Peter (1994): “Your Electronic Future". Special report. Newsweek (ed. internacional) (julio), pp. 16-35.

PÉREZ DE MENDOZA Alfredo (1987): "Las telecomunicaciones al servicio de la sociedad". Ponencia presentada en el Seminario Latinoamericano sobre el impacto socioeconómico de las nuevas tecnologías de la comunicación. México, 11 de noviembre.

PIZ Víctor Felipe (1994): “Ganó el gobierno23 mil 731 mdd por la venta de empresas en cinco años”. El Financiero, 23 de julio. México, D. F.

RAPAPORT Carla (1993): “The new US push into Europe". Fortune International Vol. 128, Núm. 16, 27 de diciembre.

SCT (s. f.): "Opportunities in the mexican telecommunications systems". En: SCT. Opportunities for investment in Mexico's basic infrastructure. (s. d.). 